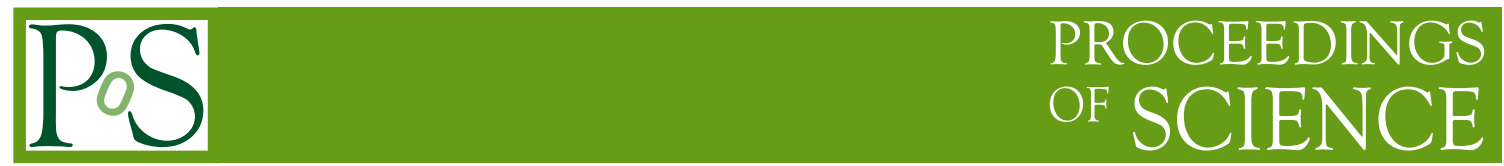

\title{
Multiple M-Branes
}

\author{
Neil Lambert* \\ Theory Division \\ Department of Physics \\ CERN \\ and \\ Department of Mathematics \\ King's College London \\ E-mail: neil.lambert@cern.ch
}

We review highly supersymmetric three-dimensional Chern-Simons-Matter conformal field theories as the worldvolume theory of multiple M2-branes and discuss some of their physical applications. We also briefly discuss the mysterious $(2,0)$ theory on multiple M5-branes.

Proceedings of the Corfu Summer Institute 2012 "School and Workshops on Elementary Particle Physics and Gravity"

September 8-27, 2012

Corfu, Greece

${ }^{*}$ Speaker. 


\section{Preface}

In these lectures we want to discuss the conformal field theories that appear on the worldvolumes of multiple M-branes. We will mainly consider M2-branes. M5-branes remain very mysterious whereas recent years has seen great progress in understanding M2-branes.

The worldvolume theories of M2-branes are certain highly supersymmetric Chern-SimonsMatter Theories (BLG and ABJM). Such theories play a similar role to Yang-Mills theories on D-branes. Indeed they can be viewed as the IR conformal fixed points of the three-dimensional Yang-Mills gauge theories on D2-branes in type IIA string theory.

From the point of supersymmetry these theories are quite interesting. In particular they have the following novel features

- Matter fields are not in the adjoint representation, but yet are related to the gauge fields by supersymmetry.

- The amount of supersymmetry is determined by the gauge group and matter representation.

These are perhaps the main reasons why it took more than 30 years between the discovery of maximally supersymmetric Yang-Mills theories and maximally supersymmetric Chern-Simonsmatter theories.

Much of these lectures follow the reviews [1] and [2], which (hopefully) contain detailed references. I will therefore not give references in these lecture notes and hope that no one takes offence. Furthermore the lectures are aimed at being pedagogical rather than historical. Another review, more related to AdS/CFT, can be found in [3], whereas [4] gives a review of integrability. In particular what follows consists of three lectures (although the final one is rather brief and speculative):

1 M2-branes and Chern-Simons-Matter Theories.

- BLG

- ABJM

- 3-algebras

2 Physical Analysis

- Vacuum Moduli Space

- Gravity Dual

- Novel Higgs reduction to D2-branes

- Monopole ('t Hooft) operators

- Hidden symmetries

3 M5-branes and the $(2,0)$ CFT

- A $(2,0)$ system

The conventions used are listed in the appendix. Lastly I would like to thank the organizers for the invitation to lecture at such a nice school. 


\section{M2-branes and Chern-Simons-Matter Theories}

So we start by considering an M2-brane along $x^{0}, x^{1}, x^{2}$. This will break the Lorentz group:

$$
S O(1,10) \rightarrow S O(1,2) \times S O(8),
$$

as well as translations along $x^{3}, \ldots, x^{10}$. It will also break half of the supersymmetries $32 \rightarrow 16$ preserving those that satisfy

$$
\Gamma_{012} \varepsilon=\varepsilon
$$

The breaking of translational invariance leads to 8 Goldstone modes $X^{I}$ which can be thought of as describing the positions of the branes. The breaking of the supersymmetries leads to Goldstino modes: $\Gamma_{012} \Psi=-\Psi$. The unbroken supersymmetries act on this spectrum. In the free case, of a single M2-brane, the supersymmetry is

$$
\begin{aligned}
\delta X^{I} & =i \bar{\varepsilon} \Gamma^{I} \Psi \\
\delta \Psi & =\partial_{\mu} X^{I} \Gamma^{\mu} \Gamma^{I} \varepsilon .
\end{aligned}
$$

\subsection{BLG}

Next we wish to see how this could work for multiple, interacting M2-branes. To this end we let the fields take values in a vector space with basis $T^{a}$ :

$$
X^{I} \rightarrow X_{a}^{I} T^{a} \quad \Psi \rightarrow \Psi_{a} T^{a} .
$$

A natural guess for supersymmetry is:

$$
\begin{aligned}
\delta X_{d}^{I} & =i \bar{\varepsilon} \Gamma^{I} \Psi_{d} \\
\delta \Psi_{d} & =\partial_{\mu} X_{d}^{I} \Gamma^{\mu} \Gamma^{I} \varepsilon-\frac{1}{3 !} X_{a}^{I} X_{b}^{J} X_{c}^{K} f_{d}^{a b c} \Gamma^{I J K} \varepsilon
\end{aligned}
$$

where $f^{a b c}{ }_{d}$ are just some sort of 'structure' constants. Now we must check that this supersymmetry algebra closes:

$$
\left[\delta_{1}, \delta_{2}\right] X_{d}^{I}=-2 i \bar{\varepsilon}_{2} \Gamma^{\mu} \varepsilon_{1} \partial_{\mu} X_{d}^{I}-\left(2 i \bar{\varepsilon}_{2} \Gamma^{J K} \varepsilon_{1} X_{a}^{J} X_{b}^{K} f_{d}^{a b c}\right) X_{c}^{I} .
$$

The first term is simply a translation by $v^{\mu}=-2 i \bar{\varepsilon}_{2} \Gamma^{\mu} \varepsilon_{1}$. This is what we expect and indeed it is already present in the free theory. The second term must be interpreted as a symmetry:

$$
\delta X_{d}^{I}=\tilde{\Lambda}_{d}^{c} X_{c}^{I}, \quad \tilde{\Lambda}_{d}^{c}=-2 i \bar{\varepsilon}_{2} \Gamma^{J K} \varepsilon_{1} X_{a}^{J} X_{b}^{K} f_{d}^{a b c} .
$$

Furthermore since $\tilde{\Lambda}_{d}^{c}$ depends on $X^{I}$ it is a local parameter and hence we must have a gauge theory.

Therefore our next step must be to introduce a gauge field for this gauge symmetry. Following the standard procedure we define

$$
D_{\mu} X_{d}^{I}=\partial_{\mu} X_{d}^{I}-\tilde{A}_{\mu}^{c}{ }_{d} X_{c}^{I}
$$


and similarly for $\Psi_{d}$. This is gauge covariant provided that

$$
\delta \tilde{A}_{\mu}^{c}{ }_{d}=\partial_{\mu} \tilde{\Lambda}_{d}^{c}+\tilde{A}_{\mu}{ }^{c}{ }_{e} \tilde{\Lambda}_{d}^{e}-\tilde{\Lambda}^{c}{ }_{e} \tilde{A}_{\mu}{ }^{e}{ }_{d},
$$

under a gauge transformation. We can also compute the field strength from $\left[D_{\mu}, D_{v}\right] X_{b}^{I}=\tilde{F}_{\mu \nu}{ }^{a}{ }_{b} X_{a}^{I}$ and find

$$
\tilde{F}_{\mu \nu b}{ }^{a}=\partial_{v} \tilde{A}_{\mu b}{ }^{a}{ }_{b}-\partial_{\mu} \tilde{A}_{v}{ }^{a} b-\tilde{A}_{\mu}{ }^{a}{ }_{c} \tilde{A}_{v}{ }^{c} b+\tilde{A}_{v}{ }^{a}{ }_{c} \tilde{A}_{\mu}{ }^{c} b .
$$

We must also postulate supersymmetry variations for and including the gauge field. Thus we are led to

$$
\begin{aligned}
\delta X_{a}^{I} & =i \bar{\varepsilon} \Gamma^{I} \Psi_{a} \\
\delta \Psi_{a} & =D_{\mu} X_{a}^{I} \Gamma^{\mu} \Gamma^{I} \varepsilon-\frac{1}{6} X_{b}^{I} X_{c}^{J} X_{d}^{K} f^{b c d}{ }_{a}^{I J K} \varepsilon \\
\delta \tilde{A}_{\mu}{ }^{b}{ }_{a} & =i \bar{\varepsilon} \Gamma_{\mu} \Gamma_{I} X_{c}^{I} \Psi_{d} f^{c d b}{ }_{a} .
\end{aligned}
$$

Indeed this closes on-shell, provided that the 'structure constants' satisfy the 'Fundamental identity':

$$
f^{a b c} f_{d}^{e f g}=f^{e f a} f_{d}^{g b c}+f_{d}^{a g c} f_{g}^{e f b}+f_{d}^{a b g} f_{g}^{e f c} .
$$

Rather than give the equations of motion that are required for closure we simply note that they come from the lagrangian

$$
\mathscr{L}=-\frac{1}{2} D_{\mu} X^{a I} D^{\mu} X_{a}^{I}+\frac{i}{2} \bar{\Psi}^{a} \Gamma^{\mu} D_{\mu} \Psi_{a}+\frac{i}{4} \bar{\Psi}_{b} \Gamma_{I J} X_{c}^{I} X_{d}^{J} \Psi_{a} f^{a b c d}-V+\mathscr{L}_{C S},
$$

where the potential is

$$
V=\frac{1}{12} X_{a}^{I} X_{b}^{J} X_{c}^{K} X_{e}^{I} X_{f}^{J} X_{g}^{K} f^{a b c d} f_{d}^{e f g}
$$

and the 'twisted' Chern-Simons term is

$$
\mathscr{L}_{C S}=\frac{1}{2} \varepsilon^{\mu v \lambda}\left(f^{a b c d} A_{\mu a b} \partial_{v} A_{\lambda c d}+\frac{2}{3} f^{c d a}{ }_{g} f^{e f g b} A_{\mu a b} A_{v c d} A_{\lambda e f}\right) .
$$

To construct the action (but not the equations of motion) we required an invariant inner-product $h^{a b}$ on the vector space with

$$
h^{d e} f_{e}^{a b c}=f^{a b c d}=f^{[a b c d]} .
$$

Thus we have constructed the BLG theory with 16 supersymmetries, an $S O(8)$ R-symmetry and conformal invariance.

But for a positive definite choice of $h^{a b}$ it turns out that there is just one finite-dimensional solution:

$$
f^{a b c d}=\frac{2 \pi}{k} \varepsilon^{a b c d} \quad a, b, c, d=1,2,3,4 .
$$

What does this look like in more familiar gauge theory terms? Well the gauge algebra generated by $\tilde{\Lambda}_{d}^{c}=\Lambda_{a b} f_{d}^{a b c}$ is simply all anti-symmetric $4 \times 4$ real matrices: $s o(4)=s u(2) \oplus s u(2)$. The two $s u(2)$ factors can be thought of as self-dual and anti-self-dual generators. The fields $X_{a}^{I}, \Psi_{a}$ are in the $\mathbf{4}=\mathbf{2}+\overline{\mathbf{2}}=$ bifundamental.

$$
\mathscr{L}_{C S}=\frac{k}{8 \pi} \varepsilon^{\mu v \lambda} \operatorname{tr}\left(\tilde{A}_{\mu}^{+} \partial_{v} \tilde{A}_{\lambda}^{+}+\frac{2}{3} \tilde{A}_{\mu}^{+} \tilde{A}_{v}^{+} \tilde{A}_{\lambda}^{+}-\tilde{A}_{\mu}^{-} \partial_{v} \tilde{A}_{\lambda}^{-}-\frac{2}{3} \tilde{A}_{\mu}^{-} \tilde{A}_{v}^{-} \tilde{A}_{\lambda}^{-}\right)
$$


where $\tilde{A}_{\mu}^{ \pm a}{ }_{b}$ are the (anti)-self-dual parts of $\tilde{A}_{\mu}{ }^{a} b$. The Chern-Simons terms are not gauge-invariant. In particular one finds that under a general gauge transformation

$$
\int d^{3} x \mathscr{L}_{C S} \rightarrow \int d^{3} x \mathscr{L}_{C S}+2 \pi k w^{+}-2 \pi k w^{-},
$$

where $w^{ \pm}$are the winding numbers of the two $S U(2)$ gauge group transformations, viewed as maps from (compactified) euclidean spacetime $S^{3}$ into $S U(2) \equiv S^{3}$. Thus we require that $k$, known as the Chern-Simons level, is an integer. Therefore there is no continuous parameter. However $1 / k$ can be thought of as a discrete coupling constant.

\section{2 АBJM}

Thats great! Its the only example of a maximally supersymmetric non-gravitational lagrangian that is not Yang-Mills. But rather limited as it turns out to only describe 2 M2's and only when $k=1,2$ (see later). To do better we need to generalize and it turns out that we should consider slightly less supersymmetry:

- $16 \rightarrow 12$ supercharges

- $S O(8)$ is reduced to $S U(4) \times U(1)$.

So one can now play a similar game but now $X_{a}^{I}$ written as 4 complex scalar fields:

- $Z_{a}^{A}$ in $\mathbf{4}$ of $S U(4)$ with $U(1)$ charge 1

- $\left(Z_{a}^{A}\right)^{\dagger}=\bar{Z}_{A}^{a}$ in $\overline{\mathbf{4}}$ of $S U(4)$ with $U(1)$ charge -1 .

For the fermions we write $\Psi_{a}$ written as 4 complex fermions

- $\psi_{A a}$ in $\overline{4}$ of $S U(4)$ with $U(1)$ charge 1

- $\left(\psi_{A a}\right)^{\dagger}=\psi^{A a}$ in 4 of $S U(4)$ with $U(1)$ charge -1 .

The 16 components of $\varepsilon$ are reduced to $\varepsilon^{A B}=-\varepsilon^{B A}$ in $\mathbf{6}$ of $S U$ (4) with $U(1)$ charge 0 .

- $\left(\varepsilon^{A B}\right)^{*}=\varepsilon_{A B}=\frac{1}{2} \varepsilon_{A B C D} \varepsilon^{C D}$.

If one follows the same steps as above one finds the correct supersymmetry transformations are

$$
\begin{aligned}
\delta Z_{d}^{A} & =i \bar{\varepsilon}^{A B} \psi_{B d} \\
\delta \psi_{B d} & =\gamma^{\mu} D_{\mu} Z_{d}^{A} \varepsilon_{A B}+f^{a b}{ }_{c d} Z_{a}^{C} Z_{b}^{A} \bar{Z}_{C}^{c} \varepsilon_{A B}+f^{a b}{ }_{c d} Z_{a}^{C} Z_{b}^{D} \bar{Z}_{B}^{c} \varepsilon_{C D} \\
\delta \tilde{A}_{\mu}{ }^{c} d & =-i \bar{\varepsilon}_{A B} \gamma_{\mu} Z_{a}^{A} \psi^{B b} f_{b d}^{c a}+i \bar{\varepsilon}^{A B} \gamma_{\mu} \bar{Z}_{A}^{b} \psi_{B a} f^{c a}{ }_{b d} .
\end{aligned}
$$

In this case the 'structure constants' satisfy

$$
\begin{aligned}
& f^{e f}{ }_{g b} f^{c b}{ }_{a d}+f^{f e}{ }_{a b} f^{c b}{ }_{g d}+f_{g a}^{*} f b f^{c e}{ }_{b d}+f_{a g}^{* e b} f^{c f}{ }_{b d}=0 \\
& \left(f^{a b}{ }_{c d}\right)^{*}=f_{a b}^{c d} \\
& f^{a b}{ }_{c d}=-f^{b a}{ }_{c d} \text {. }
\end{aligned}
$$


The invariant lagrangian is

$$
\begin{aligned}
\mathscr{L}= & -D^{\mu} \bar{Z}_{A}^{a} D_{\mu} Z_{a}^{A}-i \bar{\psi}^{A a} \gamma^{\mu} D_{\mu} \psi_{A a}-V+\mathscr{L}_{C S} \\
& -i f^{a b}{ }_{c d} \bar{\psi}^{A d} \psi_{A a} Z_{b}^{B} \bar{Z}_{B}^{c}+2 i f^{a b}{ }_{c d} \bar{\psi}^{A d} \psi_{B a} Z_{b}^{B} \bar{Z}_{A}^{c} \\
& +\frac{i}{2} \varepsilon_{A B C D} f^{a b}{ }_{c d} \bar{\psi}^{A d} \psi^{B c} Z_{a}^{C} Z_{b}^{D}-\frac{i}{2} \varepsilon^{A B C D} f^{c d}{ }_{a b} \bar{\psi}_{A c} \psi_{B d} \bar{Z}_{C}^{a} \bar{Z}_{D}^{b},
\end{aligned}
$$

where

$$
\begin{aligned}
V & =\frac{2}{3} \Upsilon_{B d}^{C D} \bar{\Upsilon}_{C D}^{B d} \\
\Upsilon_{B d}^{C D} & =f^{a b}{ }_{c d} Z_{a}^{C} Z_{b}^{D} \bar{Z}_{B}^{c}-\frac{1}{2} \delta_{B}^{C} f^{a b}{ }_{c d} Z_{a}^{E} Z_{b}^{D} \bar{Z}_{E}^{c}+\frac{1}{2} \delta_{B}^{D} f^{a b}{ }_{c d} Z_{a}^{E} Z_{b}^{C} \bar{Z}_{E}^{c},
\end{aligned}
$$

and the 'twisted' Chern-Simons term $\mathscr{L}_{C S}$ is given by

$$
\mathscr{L}_{C S}=\frac{1}{2} \varepsilon^{\mu \nu \lambda}\left(f^{a b}{ }_{c d} A_{\mu}{ }^{c} \partial_{\nu} A_{\lambda}{ }^{d}{ }_{a}+\frac{2}{3} f^{a c}{ }_{d g} f^{g e}{ }_{f b} A_{\mu}{ }^{b}{ }_{a} A_{\nu}{ }^{d}{ }_{c} A_{\lambda}{ }^{f}{ }_{e}\right) .
$$

The structure constants define a triple product:

$$
\left[T^{a}, T^{b} ; T_{c}\right]=f^{a b}{ }_{c d} T^{d} .
$$

One infinite class of examples are given by $m \times n$ complex matrices:

$$
[A, B ; C]=\frac{2 \pi}{k}\left(A C^{\dagger} B-B C^{\dagger} A\right) .
$$

Gauge group generated by $\delta Z_{d}^{A}=\Lambda^{c}{ }_{b} f^{a b}{ }_{c d} Z_{a}^{A}$ is

$$
\delta Z^{A}=M Z^{A}-Z^{A} N
$$

where $M, N$ are $m \times m$ and $n \times n$ matrices respectively. Thus the gauge group is $U(n) \times U(m)$ with matter in the bi-fundamental. Furthermore the twisted Chern-Simons term is again just a sum of Chern-Simons terms of $U(n)$ and $U(m)$ with levels $k$ and $-k$.

There is a slight caveat for the case of $m=n$. In this case the gauge group reduces to $S U(n) \times$ $S U(n)$ because the two abelian $U(1)$ factors cancel. However one can simply add the $U(1) \times U(1)$ gauge fields back in by hand to to get $U(n) \times U(n)$. These lead to

- $U(n) \times U(n) \mathrm{ABJM}$ theories

- $U(m) \times U(n) m \neq n \mathrm{ABJ}$ theories

In the special case of $S U(2) \times S U(2)$ we recover the BLG theory but written in complex notation.

In these lectures we will restrict attention to these BLG, ABJM and ABJ theories. There is an interesting story corresponding to other gauge groups and various amounts to supersymmetry. For example there is also a solution with 12 supersymmetries and group $S p(n) \times O(2)$. Still more examples can be found with less supersymmetry $(10,8, \ldots)$ using other choices of gauge groups and matter representations. 


\subsection{Three-algebras}

Before moving on to look at the physical interpretation of these theories as the worldvolume effective action for M2-branes let us look more closely at this triple product on the vector space $\mathscr{V}$ generated by $T^{a}$. This defines a 3-algebra:

$$
[\cdot, \cdot \cdot \cdot]: \mathscr{V} \otimes \mathscr{V} \otimes \overline{\mathscr{V}} \rightarrow \mathscr{V}
$$

The key idea is that the analog of adjoint map

$$
\varphi_{U, \bar{V}}(X)=[X, U ; \bar{V}] \quad \varphi_{U, \bar{V}}(\bar{X})=-[\bar{X}, \bar{V} ; U],
$$

is a derivation

$$
\varphi_{U, \bar{V}}([X, Y ; \bar{Z}])=\left[\varphi_{U, \bar{V}}(X), Y ; \bar{Z}\right]+\left[X, \varphi_{U, \bar{V}}(Y) ; \bar{Z}\right]+\left[X, Y ; \varphi_{U, \bar{V}}(\bar{Z})\right],
$$

And this is the fundamental identity that we encountered.

The fundamental identity tells us that the action of $\varphi$ on $\mathscr{V}$ is that of a lie-algebra $\mathscr{G}$ generated by $\varphi_{U, \bar{V}}$ for all $U, V \in \mathscr{V}$ i.e. $\mathscr{V}$ is representation of $\mathscr{G}$. Thus a 3 -algebra defines a lie-algebra (e.g. . $u(m) \oplus u(n)$ ) along with a preferred representation (e.g.. the bi-fundamental)

In fact the reverse is also true: Given a Lie-algebra and a representation (along with invariant inner-products) one can always construct a triple product satisfying the fundamental identity (via the so-called Faulkner map).

Thus one need not think of a 3-algebra and instead just think of the gauge group and matter representation. However the symmetry properties of the triple product naturally lead to these rather esoteric choices (and indefinite inner-products on the lie-algebra leading to the opposite levels of the Chern-Simons terms). Thus 3-algebras are the natural framework with which to discuss these supersymmetric Chern-Simons-Matter theories. Indeed the classification of such theories is essentially a classification of 3-algebras.

In particular we see that the amount of supersymmetry is determined by the symmetry properties of the triple product and determines the gauge algebra and matter representations. This should be contrast with the familiar case of super-Yang-Mills theories where the gauge algebra is arbitrary and all fields are in the adjoint (for more than 8 supersymmetries). This new situation arises because the Chern-Simons kinetic terms for the gauge field means that they do not represent propagating dynamical degrees of freedom.

\section{Physical Analysis}

Having constructed these new and highly supersymmetric three-dimensional field theories we next have to see how they are related to M2-branes.

\subsection{Vacuum Moduli Space}

The first thing to look at is the vacuum moduli space. This tells us the space of all the zeroenergy configurations of the M2-branes and hence should correspond to putting $n$ indistinguishable objects in some eight-dimensional space. Consider ABJM where the vacuum condition is

$$
\left[Z^{A}, Z^{B} ; \bar{Z}_{C}\right]=0 \Longleftrightarrow Z^{A} \bar{Z}_{C} Z^{B}=Z^{B} \bar{Z}_{C} Z^{A}
$$


Generically this implies that all the $Z^{A}$ commute (c.f. D-branes):

$$
Z^{A}=\left(\begin{array}{ccc}
z_{i}^{A} & & \\
& \ddots & \\
& & z_{n}^{A}
\end{array}\right)
$$

To see that this is all requires one to evaluate the mass formula for small fluctuations which one finds is non-zero. Why generically? there are special points where extra massless modes arise but these are expected to be lifted by non-perturbative effects.

However, as is the case with D-branes, we must identify fields that differ by gauge transformations. But in contrast to D-branes the scalar fields are not in the adjoint by in the bi-fundamental:

$$
Z^{A} \rightarrow g_{L} Z^{A} g_{R}^{-1}
$$

We could set $g_{L}=g_{R}$ so that this is an adjoint action, as with D-branes. This allows us to put $Z^{A}$ in diagonal form (as we have already done) and in addition acts as

$$
z_{i}^{A} \longleftrightarrow z_{j}^{A} \quad \text { for any } i \neq j
$$

e.g. for $i, j=1,2$ these are generated by

$$
g_{L}=g_{R}=\left(\begin{array}{ccccc}
0 & i & & \\
i & 0 & & & \\
& & 1 & & \\
& & & \ddots & \\
& & & & 1
\end{array}\right) .
$$

Together these generate the action of the symmetric group $S_{n}$ on $z_{i}^{A}$.

But unlike D-branes we also have continuous gauge transformations:

$$
z_{i}^{A} \rightarrow e^{i \theta_{i}} z_{i}^{A}
$$

which arise from taking

$$
g_{L}=g_{R}^{-1}=\left(\begin{array}{lll}
e^{i \theta_{1} / 2} & & \\
& \ddots & \\
& & e^{i \theta_{n} / 2}
\end{array}\right)
$$

The effect of these gauge transformations on the vacuum moduli space is more subtle. To elucidate their role we must examine the lagrangian restricted to the moduli $z_{i}^{A}$, including the gauge fields:

$$
\mathscr{L}=-\frac{1}{2} \sum_{i} D_{\mu} z_{i}^{A} D^{\mu} \bar{z}_{A i}+\frac{k}{4 \pi} \varepsilon^{\mu \nu \lambda} \sum_{i} A_{\mu i}^{L} \partial_{v} A_{\lambda i}^{L}-\frac{k}{4 \pi} \varepsilon^{\mu \nu \lambda} \sum_{i} A_{\mu i}^{R} \partial_{v} A_{\lambda i}^{R},
$$

where

$$
A_{\mu}^{L}=\left(\begin{array}{ccc}
A_{\mu 1}^{L} & & \\
& \ddots & \\
& & A_{\mu n}^{L}
\end{array}\right)
$$




$$
\begin{aligned}
A^{R} & =\left(\begin{array}{ccc}
A_{\mu 1}^{R} & & \\
& \ddots & \\
& & A_{\mu n}^{R}
\end{array}\right) \\
& D_{\mu} z_{i}^{A}=\partial_{\mu} z_{i}^{A}-i\left(A_{\mu i}^{L}-A_{\mu i}^{R}\right) z_{i}^{A} .
\end{aligned}
$$

Note that $z_{i}^{A}$ only couples to $B_{\mu i}=A_{\mu i}^{L}-A_{\mu i}^{R}$ and not to $Q_{\mu i}=A_{\mu i}^{L}+A_{\mu i}^{R}$ :

$$
\mathscr{L}=-\frac{1}{2} \sum_{i} D_{\mu} z_{i}^{A} D^{\mu} \bar{z}_{A i}+\frac{k}{4 \pi} \varepsilon^{\mu \nu \lambda} \sum_{i} B_{\mu i} \partial_{\nu} Q_{\lambda i}
$$

with $D_{\mu} z_{i}^{A}=\partial_{\mu} z_{i}^{A}-i B_{\mu i} z_{i}^{A}$. Next it's helpful to dualize $Q_{\mu i}$ :

$$
\begin{aligned}
\mathscr{L} & =-\frac{1}{2} \sum_{i} D_{\mu} z_{i}^{A} D^{\mu} \bar{z}_{A i}+\frac{k}{8 \pi} \varepsilon^{\mu v \lambda} \sum_{i} B_{\mu i} H_{v \lambda i}-\frac{1}{8 \pi} \varepsilon^{\mu \nu \lambda} \sigma_{i} \partial_{\mu} H_{v \lambda i} \\
& \cong-\frac{1}{2} \sum_{i} D_{\mu} z_{i}^{A} D^{\mu} \bar{z}_{A i}+\frac{k}{8 \pi} \varepsilon^{\mu v \lambda} \sum_{i} B_{\mu i} H_{v \lambda i}+\frac{1}{8 \pi} \varepsilon^{\mu \nu \lambda} \partial_{\mu} \sigma_{i} H_{v \lambda i},
\end{aligned}
$$

where $H_{v \lambda i}=\partial_{v} Q_{\lambda i}-\partial_{\lambda} Q_{v i}$. Integrating out $H_{v \lambda i}$ tells us $B_{\mu i}=-k^{-1} \partial_{\mu} \sigma_{i}$ and everything is pure gauge:

$$
\mathscr{L}=-\frac{1}{2} \sum_{i} \partial_{\mu} w_{i}^{A} \partial^{\mu} \bar{w}_{A i}
$$

where $w_{i}^{A}=e^{i \sigma_{i} / k} z_{i}^{A}$ is gauge invariant.

So what? The point is that $\sigma_{i}$ is periodic:

$$
\begin{aligned}
\int \mathscr{L}\left(\sigma_{i}+2 \pi\right)-\int \mathscr{L}\left(\sigma_{i}\right) & =-\frac{1}{4} \sum_{i} \int \varepsilon^{\mu \nu \lambda} \partial_{\mu} H_{v \lambda i} \\
& =-\frac{1}{2} \sum_{i} \int d H \\
& =-\frac{1}{2} \sum_{i} \int d F^{L}+d F^{R} \\
& \in 2 \pi \mathbb{Z} .
\end{aligned}
$$

Here we have used the Dirac quantization rule

$$
\int d F \in 2 \pi \mathbb{Z}
$$

along with the fact that $B_{i}=-k^{-1} d \sigma_{i}$ implies $d B_{i}=F_{i}^{L}-F_{i}^{R}=0$.

This periodicity of $\sigma_{i}$ means that

$$
w_{i}^{A} \cong e^{2 \pi i / k} w_{i}^{A} .
$$

Thus there is an extra orbifold action in spacetime

$$
\mathbb{R}^{8} \rightarrow \mathbb{C}^{4} / \mathbb{Z}_{k},
$$

and the vacuum moduli space is

$$
\mathscr{M}=\operatorname{Sym}^{n}\left(\mathbb{C}^{4} / \mathbb{Z}_{k}\right) .
$$


This corresponds to $n$ M2-branes in an $\mathbb{C}^{4} / \mathbb{Z}_{k}$ transverse space. Furthermore the orbifold preserves 12 supersymmetries. Placing M2-branes in such an orbifold does not break any more supersymmetries.

Let us now consider ABJ with gauge group $U(n) \times U(m), n \neq m$. We write $m=n+l$. In fact one finds that the extra $n \times l$ blocks in $Z^{A}$ must vanish. Thus the vacuum moduli space is still $\operatorname{Sym}^{n}\left(\mathbb{C}^{4} / \mathbb{Z}_{k}\right)$ and so it must still describe $n \mathrm{M} 2$ 's in $\mathbb{C}^{4} / \mathbb{Z}_{k}$. However in the ABJ models parity is broken (parity corresponds to flipping $U(n) \times U(m) \rightarrow U(m) \times U(n)$ ). The interpretation is that $l$ 'fractional' M2-branes, with charge $1 / k$, are been stuck at the fixed point. This equivalently corresponds to $l$-units of discrete torsion in the background four-form $\left(H^{4}\left(\mathbb{C}^{4} / \mathbb{Z}_{k}, \mathbb{Z}\right)=\mathbb{Z}_{k}\right)$. Since $k$ fractional branes have unit charge they can move off the fixed point and therefore we require that $l \leq k$. This leads to the following conjectures

- $U(n) \times U(n+l)$ ABJ has no vacuum for $l>k$

- $U(n) \times U(n+k) \mathrm{ABJ}$ is dual to $U(n) \times U(n) \mathrm{ABJM}$.

\subsection{Dual Geometry}

Let us look more closely at the spacetime. The orbifold acts as

$$
\left(x^{0}, x^{1}, x^{2}\right) \rightarrow\left(x^{0}, x^{1}, x^{2}\right), \quad z^{A} \rightarrow e^{2 \pi i / k} z^{A},
$$

here $z^{A}$ are complex coords for the $\mathbb{C}^{4} \cong \mathbb{R}^{8}$ spanned by $x^{3}, \ldots, x^{10}$. We next write $\mathbb{R}^{8}$ in 'spherical' coordinates

$$
d s_{\mathbb{R}^{8}}^{2}=d r^{2}+r^{2} d s_{S^{7}}^{2},
$$

and then $S^{7}$ as a Hopf fibration over $\mathbb{C} P^{3}$ :

$$
d s_{S^{7}}^{2}=(d \phi+\omega)^{2}+d s_{\mathbb{C} P^{3}}^{2} .
$$

In these coordinates the orbifold simply acts on the fibre as

$$
\phi \rightarrow \phi+2 \pi i / k .
$$

At large $k$, which is weak coupling, the orbifold shrinks the fibre and we have a type IIA background.

Let use consider the large $n$ limit. The supergravity solution solution is

$$
\begin{aligned}
d s_{11}^{2} & =H^{-2 / 3}\left(-\left(d x^{0}\right)^{2}+\left(d x^{1}\right)^{2}+\left(d x^{2}\right)^{2}\right)+H^{1 / 3} d s_{\mathbb{C}^{4} / \mathbb{Z}_{k}}^{2} \\
& =\frac{r^{4}}{n^{\frac{2}{3}} r_{0}^{4}}\left(-\left(d x^{0}\right)^{2}+\left(d x^{1}\right)^{2}+\left(d x^{2}\right)^{2}\right)+\frac{n^{\frac{1}{3}} r_{0}^{2}}{r^{2}} d r^{2}+n^{\frac{1}{3}} r_{0}^{2} d s_{S^{7} / \mathbb{Z}_{k}}^{2} \\
& =\frac{n^{\frac{1}{3}} r_{0}^{2}}{4}\left(\frac{-\left(d x^{0}\right)^{2}+\left(d x^{1}\right)^{2}+\left(d x^{2}\right)^{2}+d z^{2}}{z^{2}}\right)+n^{\frac{1}{3}} r_{0}^{2} d s_{S^{7} / \mathbb{Z}_{k}}^{2},
\end{aligned}
$$

where

$$
H=1+\frac{n r_{0}^{6}}{r^{6}} .
$$


Therefore we obtain, in the large $k$ limit, an $A d S_{4} \times \mathbb{C} P^{3}$ dual type IIA geometry:

$$
d s_{10}^{2}=\frac{\sqrt{n} r_{0}^{3}}{4 k} d s_{A d S_{4}}^{2}+\frac{\sqrt{n} r_{0}^{3}}{k^{3}} d s_{\mathbb{C} P^{3}}^{2},
$$

with $e^{2 \phi}=\sqrt{n} r_{0}^{3} k^{-3}, C^{(1)}=k \omega$.

\subsection{Novel Higg's Reduction to D2-branes}

Next we consider a bunch of M2-branes located at $z^{4}=i v$, far from the origin.

$$
Z^{A}=i v \delta^{A 4}+\frac{1}{\sqrt{2}} X^{A}+i \frac{1}{\sqrt{2}} X^{A+4} .
$$

If we just look at small fluctuations (small compared to the distance $v$ from the origin) then the spacetime, which is really $\mathbb{R}^{8} / \mathbb{Z}_{k}$, looks like $\mathbb{R}^{7} \times\left(S^{1} / \mathbb{Z}_{k}\right)$.

This leads to a 'novel Higgs' effect since $v \neq 0$ breaks $U(n) \times U(n) \rightarrow U(n)$. However rather than a massless gauge field becoming massive the starting point is a non-dynamical gauge field and the result is a dynamical one (recall that in three-dimensions a massless gauge field has just one degree of freedom).

We won't give the calculation in detail here. Rather we note that $B_{\mu}=A_{\mu}^{L}-A_{\mu}^{R}$ has no kinetic term and can integrated out. At lowest order this is quite straightforward and yields a familiar Yang-Mills kinetic term for $A_{\mu}$. In this process $A_{\mu}=A_{\mu}^{L}+A_{\mu}^{R}$ eats $X^{8}$ and becomes dynamical.

The resulting action has a dynamical vector and 7 scalars (plus fermions) and must preserve at least 16 supersymmetries. Therefore it must be, at least at leading order, three-dimensional maximally supersymmetric Yang-Mills:

$$
\mathscr{L}=\frac{1}{g_{Y M}^{2}} \mathscr{L}_{M S Y M}+\mathscr{O}\left(1 / v^{3}\right),
$$

where $g_{Y M}^{2}=8 \pi^{2} v^{2} / k$. The higher order corrections correspond to the fact that finite fluctuations sense that spacetime is not $\mathbb{R}^{7} \times S^{1}$ but $\mathbb{R}^{8} / \mathbb{Z}_{k}$. Thus we have recovered the link to D2-branes.

\subsection{Monopole ('t Hooft) operators and hidden symmetries}

Let us return to the moduli space. It follows that we can think of

$$
Z^{A}=\left(\begin{array}{ccc}
z_{i}^{A} & & \\
& \ddots & \\
& & z_{n}^{A}
\end{array}\right)
$$

as describing the positions of $n$ M2-branes in $\mathbb{C}^{4} / \mathbb{Z}_{k}$. Furthermore the natural circle for the Mtheory direction is the over-all phase. Suppose we wanted to describe $n$ M2-branes moving along the M-theory circle, possibly with different speeds. One might expect that this corresponds to

$$
Z^{A}=\left(\begin{array}{ccc}
z_{i}^{A} e^{i \omega_{1} t} & & \\
& \ddots & \\
& & z_{n}^{A} e^{i \omega_{n} t}
\end{array}\right)
$$


but this is pure gauge! We can un-do it by taking

$$
g_{L}=g_{R}^{-1}=\left(\begin{array}{lll}
e^{-i \omega_{1} t / 2} & & \\
& \ddots & \\
& & e^{-i \omega_{n} t / 2}
\end{array}\right) .
$$

Note that this gauge transformation is not allowed for D-branes where the scalars are in the adjoint. So how do the M2-branes 'explore' the full transverse space?

Let us set the fermions to zero and construct the hamiltonian

$$
\begin{aligned}
H= & \operatorname{tr} \int d^{2} x \Pi_{Z^{A}} \Pi_{\bar{Z}_{A}}+D_{i} Z^{A} D^{i} \bar{Z}_{A}+V \\
& +\left(i Z^{A} \Pi_{Z^{A}}-i \Pi_{\bar{Z}_{A}} \bar{Z}_{A}-\frac{k}{2 \pi} F_{12}^{L}\right) A_{0}^{L} \\
& +\left(i \bar{Z}_{A} \Pi_{\bar{Z}_{A}}-i \Pi_{Z^{A}} Z^{A}+\frac{k}{2 \pi} F_{12}^{R}\right) A_{0}^{R} .
\end{aligned}
$$

As usual the time-components of the gauge field give constraints:

$$
\begin{aligned}
\frac{k}{2 \pi} F_{12}^{L} & =i Z^{A} \Pi_{Z^{A}}-i \Pi_{\bar{Z}_{A}} \bar{Z}_{A} \\
\frac{k}{2 \pi} F_{12}^{R} & =i \Pi_{Z^{A}} Z^{A}-i \bar{Z}_{A} \Pi_{\bar{Z}_{A}} .
\end{aligned}
$$

Consider the vacuum moduli again:

$$
Z^{A}=\left(\begin{array}{ccc}
\frac{1}{\sqrt{2}} R_{1}^{A} e^{i \theta_{1}^{A}} & & \\
& \ddots & \\
& & \frac{1}{\sqrt{2}} R_{n}^{A} e^{i \theta_{n}^{A}}
\end{array}\right)
$$

The constraint is

$$
\frac{k}{2 \pi} F_{12}^{L}=\frac{k}{2 \pi} F_{12}^{R}=\left(\begin{array}{lll}
\sum_{A}\left(R_{1}^{A}\right)^{2} \partial_{0} \theta_{0}^{A} & & \\
& \ddots & \\
& & \sum_{A}\left(R_{n}^{A}\right)^{2} \partial_{0} \theta_{n}^{A}
\end{array}\right) .
$$

In other words the momentum around the M-theory circle is given by the magnetic flux. This is, in spirit, the same as dualization:

$$
\partial_{\mu} X^{10}=\frac{1}{2} \varepsilon_{\mu \nu \lambda} F^{v \lambda} \quad \Longleftrightarrow \quad \partial_{0} X^{10}=F_{12} .
$$

This raises the next question: how do we compute quantities with 11D momentum. In particular the gauge invariant observables appear to only carry vanishing $U(1)$ charge:

$$
\begin{aligned}
\mathscr{O} & =\operatorname{tr}\left(Z^{A} \bar{Z}_{B} Z^{C} \ldots\right) & & \text { gauge invariant } \\
\mathscr{O} & =\operatorname{tr}\left(Z^{A} Z^{B} Z^{C} \ldots\right) & & \text { not gauge invariant } .
\end{aligned}
$$

Thus the most simple operators don't really explore all 11 dimensions. 
This brings us to monopole or 't Hooft operators: We want to create states or operators (these two are essentially the same in a CFT) that carry magnetic charge. These operators are defined as a prescription for computing correlators in the path-integral. They are not constructed as a local expression of the fields.

$$
<\mathscr{M}(y) \mathscr{O}(z) \ldots>=\int_{\oint_{y} F=2 \pi Q_{M}} D Z D \psi D A \mathscr{O}(z) e^{-S} .
$$

In other words we require the fields in the path integral to have a specific singularity

$$
F=\star \frac{Q_{M}}{2} d\left(\frac{1}{|x-y|}\right)+\text { nonsingular },
$$

where $Q_{M} \in u(n) \times u(n)$ is the magnetic flux and is subject to the standard Dirac quantization condition

$$
e^{2 \pi i Q_{M}}=1 .
$$

There is a famous result of Goddard, Nuyts and Olive that the set of $Q_{M}$ which satisfy this, modulo gauge transformations, are in one-to-one correspondence with highest weights of representations of the dual gauge group (Langlands dual in modern parlance). This can lead to complications but for us the dual of $U(n)$ is just $U(n)$. We can therefore group together various choices of fluxes into states associated with those of a representation of $U(n)$.

Furthermore we will be interested in supersymmetric monopole operators where the fields near the insertion point are those of a supersymmetric Dirac monopole (so the scalars also have a singularity).

Next we note that due to the Chern-Simons term monopole operators transform locally under a gauge transformation $\delta A_{\mu}^{L / R}=D_{\mu} \omega_{L / R}$ (with $\omega \rightarrow 0$ at infinity) as

$$
\begin{aligned}
\mathscr{M}_{Q_{M}}(x) & \rightarrow e^{(i k / 2 \pi) \operatorname{tr} \int\left(D \omega_{L} \wedge F^{L}-D \omega_{R} \wedge F^{R}\right)} \mathscr{M}_{Q_{M}}(x) \\
& =e^{i k \operatorname{tr}\left(\left(\omega_{L}(x)-\omega_{R}(x)\right) Q_{M}\right)} \mathscr{M}_{Q_{M}}(x) .
\end{aligned}
$$

Note that by construction we have broken the gauge group to $U(1)^{n} \times U(1)^{n}$. This is enough to tell us that under full gauge transformations the monopole operators transform in the representation of $U(n) \times U(n)$ whose highest weight is

$$
\vec{\Lambda}=k\left(\vec{Q}_{M},-\vec{Q}_{M}\right)
$$

(actually because of the sign the second factor is the lowest weight).

This is all very abstract (and tricky to calculate with). Consider the abelian case for example as appeared in the moduli space discussion (and Wick rotated)

$$
\mathscr{L}=-\frac{1}{2} \sum_{i} D_{\mu} z_{i}^{A} D^{\mu} \bar{z}_{A i}+\frac{k}{8 \pi} \varepsilon^{\mu \nu \lambda} \sum_{i} B_{\mu i} H_{v \lambda i}-\frac{i}{8 \pi} \varepsilon^{\mu \nu \lambda} \sigma_{i} \partial_{\mu} H_{v \lambda i}
$$

Here the monopole operators do have a local expression and are simply given by

$$
\mathscr{M}_{i}(y)=e^{i \sigma_{i}(y)} .
$$


To see this consider

$$
\begin{aligned}
<\mathscr{M}_{i}(y) \mathscr{O}(z) \ldots> & =\int D z D B D Q e^{i \sigma_{i}(y)} \mathscr{O}(z) e^{-\int d^{3} x \mathscr{L}(x)} \\
& =\int D z D B D Q \mathscr{O}(z) e^{-\int d^{3} x \mathscr{L}(x)-i \sigma_{i}(x) \delta(x-y)}
\end{aligned}
$$

This is the same as taking

$$
\frac{1}{8 \pi} \varepsilon^{\mu \nu \lambda} \partial_{\mu} H_{v \lambda i} \rightarrow \frac{1}{8 \pi} \varepsilon^{\mu \nu \lambda} \partial_{\mu} H_{v \lambda i}+8 \pi \delta(x-y),
$$

i.e. inserting a magnetic charge at $x=y$.

Thus our gauge invariant operator on the moduli space is just

$$
w_{i}^{A}=e^{i \sigma_{i} / k} z_{i}^{A}=\left(\mathscr{M}_{i}\right)^{\frac{1}{k}} z_{i}^{A},
$$

and indeed $\mathscr{M}_{i}$ has charge $(k,-k)$ under the appropriate $U(1) \times U(1)$ factor.

Classically $e^{i \sigma_{i}}$ has scaling dimension zero (because $B_{i} \propto d \sigma_{i}$ has scaling dimension one). What about quantum mechanically? You might think this was shifted because of normal ordering (c.f. $e^{i k X}$ on the string worldsheet). However the momentum conjugate to $B_{\mu i}$ is $A_{\mu i}$ and thus $B_{\mu i}$ and hence $\sigma_{i}$ has vanishing OPE with itself. So no normal ordering does not cause any anomalous dimension and $e^{i \sigma_{i}}$ is dimension zero in the quantum theory. It is expected that monopole operators have vanishing conformal dimension in the full ABJM and BLG theories.

\subsection{Hidden symmetries}

Let us return to the maximally supersymmetric cases $k=1,2$ where the transverse space is

$$
k=1: \mathbb{R}^{8}, \quad k=2: \mathbb{R}^{8} / \mathbb{Z}_{2} .
$$

M2-branes in these backgrounds preserve 16 supersymmetries. What happened to the extra two supersymmetries and $S O(8)$ R-symmetry? The claim is that they are there but just not manifest (this is okay since $k=1,2$ is strongly coupled and the classical intuition based on the lagrangian could be misleading).

The extra supersymmetry comes from the supercurrent

$$
J_{\mu}=\mathscr{M}^{a b} D_{\mu} Z_{a}^{A} \psi_{A b}
$$

and extra R-symmetries from

$$
J_{\mu}^{A B}=\mathscr{M}^{a b}\left(Z_{a}^{A} D_{\mu} Z_{b}^{B}-Z_{a}^{B} D_{\mu} Z_{b}^{A}+i \varepsilon^{A B C D} \bar{\psi}_{C a} \gamma_{\mu} \psi_{D b}\right),
$$

Recall that $Z^{A}$ and $\psi_{A}$ are both in the $(\mathbf{n}, \overline{\mathbf{n}})$, so $D_{\mu} Z^{A} \psi_{A}, Z^{A} D_{\mu} Z^{B}$ and $\bar{\psi}_{C} \psi_{D}$ are not gauge invariant. In particular they are in the tensor product of two fundamental representations of the $U(n) \times U(n)$ with $U(1)$ charge 2 .

So for these currents to exist we need to dress them up with a monopole operator $\mathscr{M}^{a b}$ is in the tensor product of two anti-bi-fundamental representations of $U(n) \times U(n)$ with $U(1)$ charge -2 . When does such an $\mathscr{M}$ exist? 
The (highest weight,lowest weight) for two-tensored anti-bi-funamental representation is

$$
\vec{\Lambda}=\left(2 \vec{\lambda}_{n-1},-2 \vec{\lambda}_{n-1}\right) .
$$

According to our discussion about monopole operators we must have

$$
\vec{\Lambda}=k\left(\vec{Q}_{M},-\vec{Q}_{M}\right)
$$

for a monopole charge vector $\vec{Q}_{M}$. Thus $\mathscr{M}$ only exists if

- $k=1, \vec{Q}_{M}=2 \vec{\lambda}_{n-1}$

- $k=2, \vec{Q}_{M}=\vec{\lambda}_{n-1}$.

But this is precisely as required! Thus it is consistent to conjecture that the ABJM theories at $k=1,2$ do have enhanced symmetry and supersymmetry. For two M2-branes we can make these manifest as we now see.

Let us now return to where we started: BLG. This is an $S U(2) \times S U(2)$ Chern-Simons-Matter theory with maximal supersymmetry and an $S O(8)$ R-symmetry. How does it fit in? One point to realise is that in addition to the choice of $k$ there is also a choice of the global gauge group: $S U(2) \times S U(2)$ or $(S U(2) \times S U(2)) / \mathbb{Z}_{2}$. Among other things this choice affects the vacuum moduli space since it alters the Dirac quantization condition. To cut a long(ish) story short one finds:

- $\operatorname{BLG}(S U(2) \times S U(2)) / \mathbb{Z}_{2}$ at $k=1$ is dual to $\operatorname{ABJM} U(2) \times U(2)$ at $k=1$, i.e. $2 \mathrm{M} 2$ 's in $\mathbb{R}^{8}$

- $\mathrm{BLG} S U(2) \times S U(2)$ at $k=2$ is dual to $\operatorname{ABJM} U(2) \times U(2)$ at $k=2$, i.e. $2 \mathrm{M} 2$ 's in $\mathbb{R}^{8} / \mathbb{Z}_{2}$

- $\operatorname{BLG}(S U(2) \times S U(2)) / \mathbb{Z}_{2}$ at $k=4$ is dual to $\mathrm{ABJ} U(2) \times U(3)$ at $k=2$, i.e. $2 \mathrm{M} 2$ 's in $\mathbb{R}^{8} / \mathbb{Z}_{2}$ with one fractional brane.

Thus the original BLG theory describes $2 \mathrm{M} 2$-branes in $\mathbb{R}^{8}$ or $\mathbb{R}^{8} / \mathbb{Z}_{2}$ with the benefit, in contrast to $\mathrm{ABJM}$, that all symmetries are manifest.

\section{M5-branes and the $(2,0)$ CFT}

Can we try our luck with M5-branes? This is a notoriously difficult problem (but then again the same was once said of M2-branes). However it really does seem hard since we are now talking about six-dimensional conformal field theories and there are no known good quantum theories above four dimensions. Nevertheless M-theory (as well as other arguments) tells us that such a theory exists, without gravity. It must be a very rich and novel theory dual to $A d S_{7} \times S^{4}$. Its symmetries and fields can be deduced from the breaking

$$
S O(1,10) \rightarrow S O(1,5) \times S O(5)
$$


The preserved supersymmetries sastistfy

$$
\Gamma_{012345} \varepsilon=\varepsilon,
$$

leading to $(2,0)$ supersymmetry in six-dimensions.

The multiplet contains 5 scalars $X^{I}, I=6,7,8,9,10$, corresponding to the breaking of translational invariance, and fermions which satisfy $\Gamma_{012345} \Psi=-\Psi$ and arise from the breaking of supersymmetry. Since 5 scalar degrees of freedom cannot on their own form the bosonic part of a supermultiplet with 16 supersymmetries we require 3 more bosonic degrees of freedom. A massless vector field would have 4 degrees of freedom so that won't do. A two-form, with a three-form field strength has six degrees of freedom. That's also too much but we can impose that the field strength is self-dual which brings us back to 3 degrees of freedom. And this is indeed the right answer.

The free supersymmetry transformations are ${ }^{1}$

$$
\begin{aligned}
\delta X^{I} & =i \bar{\varepsilon} \Gamma^{I} \Psi \\
\delta \Psi & =\Gamma^{\mu} \Gamma^{I} \partial_{\mu} X^{I} \varepsilon+\frac{1}{3 !} \frac{1}{2} \Gamma^{\mu \nu \lambda} H_{\mu \nu \lambda} \varepsilon \\
\delta H_{\mu \nu \lambda} & =3 i \bar{\varepsilon} \Gamma_{[\mu \nu} \partial_{\lambda]} \Psi,
\end{aligned}
$$

and the equations of motion are those of free fields with $d H=0$ (and hence $d H=d \star H=0$ ). These equations can be reduced to the D4-brane of type IIA string theory if one sets $\partial_{5}=0$ and

$$
F_{\mu v}=H_{\mu v 5} .
$$

\subsection{A $(2,0)$ System}

We wish to generalise this algebra to non-abelian fields. Following as we did for M2-branes we introduce a covariant derivative

$$
D_{\mu} X_{a}^{I}=\partial_{\mu} X_{a}^{I}-\tilde{A}_{\mu a}^{b} X_{b}^{I} .
$$

However in order to introduce interactions, which appear in the formula for $\delta \Psi_{a}$ we require a terms with an odd number of $\Gamma_{\mu}$ matrices. In particular we'd like a term of the form $X_{a}^{I} X_{b}^{J} \Gamma_{\mu} \Gamma^{I J}$. To soakup the spare $\mu$ index we are forced to introduce a new field $C_{a}^{\mu}$. Looking for the most general set of supersymmetry transformations leads to

$$
\begin{aligned}
\delta X_{a}^{I} & =i \bar{\varepsilon} \Gamma^{I} \Psi_{a} \\
\delta \Psi_{a} & =\Gamma^{\mu} \Gamma^{I} D_{\mu} X_{a}^{I} \varepsilon+\frac{1}{12} \Gamma_{\mu \nu \lambda} H_{a}^{\mu \nu \lambda} \varepsilon-\frac{1}{2} \Gamma_{\lambda} \Gamma^{I J} C_{b}^{\lambda} X_{c}^{I} X_{d}^{J} f^{c d b}{ }_{a} \varepsilon \\
\delta H_{\mu \nu \lambda a} & =3 i \bar{\varepsilon} \Gamma_{[\mu \nu} D_{\lambda]} \Psi_{a}+i \bar{\varepsilon} \Gamma^{I} \Gamma_{\mu \nu \lambda{ }_{\kappa}} C_{b}^{\kappa} X_{c}^{I} \Psi_{d} f^{c d b}{ }_{a} \\
\delta \tilde{A}_{\mu a}^{b} & =i \bar{\varepsilon} \Gamma_{\mu \lambda} C_{c}^{\lambda} \Psi_{d} f^{c d b}{ }_{a} \\
\delta C_{a}^{\mu} & =0
\end{aligned}
$$

where $f^{a b c}{ }_{d}$ are totally anti-symmetric structure constants of a (possibly Lorentzian) 3-algebra. This algebra closes with the on-shell conditions

$$
0=D^{2} X_{a}^{I}-C_{b}^{v} C_{v g} X_{c}^{J} X_{e}^{J} X_{f}^{I} f^{e f g} f^{c d b}{ }_{a}-\frac{i}{2} \bar{\Psi}_{c} C_{n}^{v} \Gamma_{\nu} \Gamma^{I} \psi_{d} f^{c d b}{ }_{a}
$$

\footnotetext{
${ }^{1}$ Note that in this lecture we use $\mu v=01,2, . ., 5$ and $I, J=6,7,8 \ldots 10$.
} 


$$
\begin{aligned}
0 & =D_{[\mu} H_{v \lambda \rho] a}+\frac{1}{4} \varepsilon_{\mu v \lambda \rho \sigma \tau} C_{b}^{\sigma} X_{c}^{I} D^{\tau} X_{d}^{I} f^{c d b}{ }_{a}+\frac{i}{8} \varepsilon_{\mu v \lambda \rho \sigma \tau} C_{b}^{\sigma} \bar{\Psi}_{c} \Gamma^{\tau} \psi_{d} f^{c d b}{ }_{a} \\
0 & =\Gamma^{\mu} D_{\mu} \Psi_{a}+X_{c}^{I} C_{b}^{v} \Gamma_{v} \Gamma^{I} \Psi_{d} f^{c d b}{ }_{a} \\
0 & =\tilde{F}_{\mu v}{ }_{a}-C_{c}^{\lambda} H_{\mu v \lambda d} f^{c d b}{ }_{a} \\
0 & =D_{\mu} C_{a}^{v}=C_{c}^{\mu} C_{d}^{v} f^{c d b}{ }_{a} \\
0 & =C_{c}^{\rho} D_{\rho} X_{d}^{I} f^{c d b}{ }_{a}=C_{c}^{\rho} D_{\rho} \Psi_{d} f^{c d b}{ }_{a}=C_{c}^{\rho} D_{\rho} H_{\mu \nu \lambda a} f^{c d b}{ }_{a} .
\end{aligned}
$$

Thus we have found a system of equations with $(2,0)$ supersymmetry, $\mathrm{SO}(5) \mathrm{R}$-symmetry and scale symmetry ( $C_{a}^{\mu}$ has dimensions of length). We note that this construction would also work if we did not put an algebra index on $C^{\mu}$, in which case the 3-algebra structure constants reduce to those of a lie-algebra: $f^{a b}{ }_{c}$.

We see that $C_{a}^{\mu}$ is non-dynamical and simply picks out a fixed direction in space and in the 3-algebra and $C_{a}^{\mu} D_{\mu}=0$. The components of the fields parallel to $C_{a}^{\mu}$ in the three-algebra reduce to the free six-dimensional multiplet above. The remaining non-abelian and interacting part of the equations only depend on a reduced number of coordinates. In particular if $C^{\mu}$ is chosen to be spacelike then the equation of motion become those of five-dimensional maximally supersymmetric Yang-Mills (one also finds a curious real and euclidean form of five-dimensional maximally supersymmetric Yang-Mills if $C^{\mu}$ is timelike). If $C^{\mu}$ is taken to be null then the equations of motion reduce to one-dimensional motion on the moduli space of self-dual four-dimensional gauge fields (with the other null coordinate 'perpendicular' to $C^{\mu}$ playing the role of time).

Nevertheless the system is six-dimensional. To see this we can construct a conserved energymomentum tensor

$$
\begin{aligned}
T_{\mu v}= & D_{\mu} X_{a}^{I} D_{v} X^{I a}-\frac{1}{2} \eta_{\mu v} D_{\lambda} X_{a}^{I} D^{\lambda} X^{I a} \\
& +\frac{1}{4} \eta_{\mu v} C_{b}^{\lambda} X_{a}^{I} X_{c}^{J} C_{\lambda g} X_{f}^{I} X_{e}^{J} f^{c d b a} f^{e f g}+\frac{1}{4} H_{\mu \lambda \rho a} H_{v}{ }^{\lambda \rho a} \\
& -\frac{i}{2} \bar{\Psi}_{a} \Gamma_{\mu} D_{v} \Psi^{a}+\frac{i}{2} \eta_{\mu v} \bar{\Psi}_{a} \Gamma^{\lambda} D_{\lambda} \Psi^{a}-\frac{i}{2} \eta_{\mu v} \bar{\Psi}_{a} C_{b}^{\lambda} X_{c}^{I} \Gamma_{\lambda} \Gamma^{I} \Psi_{d} f^{a b c d} .
\end{aligned}
$$

Here we see that the system is six-dimensional in the sense that all $P_{\mu}$ are non-vanishing. Furthermore its superalgebra is a $(2,0)$ superalgebra. One sees that the momentum parallel to $C^{\mu}$ is of the form

$$
C^{\mu} P_{\mu}=\int d^{5} x C^{\mu} T_{0 \mu} \sim \operatorname{Tr} \int F \wedge F \in \mathbb{Z},
$$

i.e. the instanton number of the gauge field. This suggest that one direction, the M-theory direction, is compact (i.e. $\mathbb{R}^{5} \times S^{1}$ ). Correspondingly the M-theory momentum is 'topological and quantized' - just as with M2-branes - and solitonic states are needed to carry the extra momentum.

This leads to the conjecture, or hope, that five-dimensional maximally supersymmetric YangMills is exactly the M5-brane theory on a circle. This seems crazy as the latter is divergent and nonrenormalizable but the former is a well-defined field theory. One resolution to this contradiction is to note that the non-perturbative sector cannot be readily disentangled from the perturbative sector. The usual argument states that solitons are suppressed by factors of

$$
e^{-1 / g_{Y M}^{2}}
$$


and hence do not appear in a perturbative expansion. However in five-dimensional super-YangMills $g_{Y M}^{2}$ has dimensions of length so we in fact must have

$$
e^{-d / g_{Y M}^{2}}
$$

where $d$ is a length-scale. Therefore there is no decoupling from perturbative dynamics if $d \leq$ $\mathscr{O}\left(g_{Y M}^{2}\right)$. Furthermore integrating over $d$, as might be expected to happen to internal states appearing in an amplitude, produces positive powers of $g_{Y M}^{2}$. Thus it is possible that five-dimensional maximally supersymmetric Yang-Mills is well-defined but only if treated non-perturbatively.

\section{Appendix: Some Conventions}

Metric:

$$
\eta=\left(\begin{array}{llll}
-1 & & \\
& 1 & & \\
& & \ddots & \\
& & & 1
\end{array}\right)
$$

Indices:

$$
\begin{aligned}
m, n \ldots & =0,1,2, \ldots 10 \\
\mu, v, \ldots & =0,1,2 \\
I, J \ldots & =3,4, \ldots, 10 \\
A, B & =1,2,3,4 .
\end{aligned}
$$

(Except in lecture 3 where $\mu, v=0,1,2 \ldots, 5$ and $I, J \ldots=6, \ldots, 10$.)

Spinors:

32 Component:

$\Gamma_{m}$ real, $\quad C=\Gamma_{0}, \quad \Psi$ real $, \quad \bar{\Psi}=\Psi^{T} C, \quad \Gamma_{012} \Psi=-\Psi$.

2 Component:

$$
\gamma_{\mu} \text { real, } \quad C=\gamma_{0}, \quad \psi_{A} \text { complex, } \quad \bar{\psi}^{A}=\psi_{A}^{\dagger} C .
$$

\section{References}

[1] J. Bagger, N. Lambert, S. Mukhi and C. Papageorgakis, arXiv:1203.3546 [hep-th].

[2] N. Lambert, Ann. Rev. Nucl. Part. Sci. 62 (2012) 285 [arXiv:1203.4244 [hep-th]].

[3] I. R. Klebanov and G. Torri, Int. J. Mod. Phys. A 25 (2010) 332 [arXiv:0909.1580 [hep-th]].

[4] T. Klose, Lett. Math. Phys. 99, 401 (2012) [arXiv:1012.3999 [hep-th]]. 